\title{
Selective looking and the noticing of unexpected events
}

\author{
ROBERT BECKLEN \\ Swarthmore College, Swarthmore, Pennsylvania \\ and \\ DANIEL CERVONE \\ Stanford University, Stanford, California
}

\begin{abstract}
Subjects in a selective-looking paradigm (Neisser \& Becklen, 1975) attended to one of two visually superimposed videotaped ballgames by responding every time the ball was passed in the target game. An unexpected, yet highly visually conspicuous, event, occurring about halfway through the 1-min game sequence, was noticed by only 18 of 85 subjects. Noticing was unrelated to the delay between the event and the posttrial inquiry, and explicit "iconic" instructions to describe the last image seen immediately after interruption proved ineffective in enhancing noticing rates, despite optimal visual conditions. Instead, noticing appeared to be related to the specific anticipatory possibilities within the attended sequence itself. Content analysis indicated that the latter part of the unexpected event sequence afforded greater anticipatory opportunities, and the findings suggested that noticers who were skilled at the main task were more likely to detect the event during this part, whereas unskilled noticers showed no such pattern. Results were consistent with and interpreted in terms of Neisser's (1976) notion of the "perceptual cycle."
\end{abstract}

In a selective-looking task, a subject is presented with two videotaped or filmed naturalistic episodes simultaneously, in full superimposition, and is asked to attend selectively to one and to ignore the other. Neisser and Becklen (1975) found that, even with minimal practice, subjects were able to accomplish this task with high accuracy. The unecological nature of the visual superimposition makes this, in principle, different from similar findings in selective listening. (For reviews, see Kahneman, 1973, and Neisser, 1967.) Auditory jumbling is not unusual in the natural environment; consequently, one might expect successful performance on selectivelistening tasks to reflect the operation of highly practiced attentional mechanisms, developed to handle situations of precisely this kind. Visual superimposition, on the contrary, hardly ever occurs naturally, and successful selection, if at all possible, ought to require considerably more practice than would be required in the auditory case. The apparent ease with which selective looking is accomplished led Neisser and Becklen to speculate that

This study was supported in part by unrestricted research funds from the Department of Psychology, Oberlin College, and in part by a research grant to the first author from Oberlin College. The support is gratefully acknowledged. The authors also wish to express thanks to Hans Wallach, Ulric Neisser, and Margaret McMahon, who commented on an earlier version of this paper. Requests for reprints should be directed to Robert Becklen, Department of Psychology, Sarah Lawrence College, Bronxville, New York 10708. special attentional mechanisms for filtering or attenuating unwanted information may not be involved at all. Rather, as long as the information required to follow the main episode is available (i.e., as long as it is not physically masked or erased by the unattended episode), subjects need simply to use their normal pickup strategies to be successful at the task (see also Neisser, 1976, for an elaboration of this argument). In a later study (Becklen, Neisser, \& Littman, Note 1; also described in Neisser, 1979), we demonstrated that kinetic features alone can provide a basis for such selection in a situation in which no information exists, in a static view, to tell the episodes apart.

A corollary issue to the question of how attentional selection takes place concerns the fate of unattended materials. This is the issue to which the present paper is primarily directed. On the basis of a "filter" theory (e.g., Broadbent, 1958), one would expect to find no evidence of availability of meaningful contents from unattended episodes, unless subjects are able to switch their attention rapidly back and forth between information sources. Likewise, on the basis of a directpickup theory, as advocated by Neisser and Becklen (1975), unattended information would be expected to have a psychological impact only to the extent that the subject actively seeks it out by, for example, taking advantage of redundancies or anticipatory possibilities in the main attended episode. That people are highly adept at developing such strategies with practice has 
been shown in studies in which they are expressly taught to perform two initially incompatible tasks simultaneously (e.g., Spelke, Hirst, \& Neisser, 1976).

There is a body of literature, however, that suggests that the analysis of unattended information is normally far more complete than either of the above positions would predict. Deutsch and Deutsch (1963) and Norman $(1968,1976)$ proposed that the process of attentional selection does not operate until after full semantic analysis of all incoming information has already taken place. Consequently, the psychological traces of unattended materials should be detectable if tested in an optimal fashion. Three primary strategies for gathering empirical support for this idea have been used: (1) testing for conscious recollection of unexpected or subjectively significant information that might be expected to make a special impression (e.g., Moray, 1959), (2) probing for memory of unattended material very shortly after its presentation (Norman, 1969), or (3) searching for some unconscious alteration in responding, such as interpretation biasing (Mackay, 1973) or shifts in autonomic responding that can be linked to the semantic contents of the unattended information (Corteen \& Wood, 1972; von Wright, Anderson, \& Stenman, 1975; but see Wardlaw \& Kroll, 1976, for a replication failure).

Within the selective-looking paradigm, a version of the first technique has been used in several studies. Neisser and Becklen (1975) found that "odd" or unexpected events in the unattended episode were noticed surprisingly rarely by naive subjects. In a later study, Neisser and Dube (Note 2) put the question to a more systematic test. Their subjects "followed" a ball game, of the kind used by Neisser and Becklen, by pushing a microswitch button each time a pass occurred. Superimposed on the attended ball game was another, similar, game in which the same three individuals appeared, wearing differently colored shirts. This featural difference, plus the coherence and continuity of the separate motion patterns in the two games, enabled subjects successfully to select one of the games with minimal or no practice. In one segment, a woman carrying a large umbrella suddenly emerged on the screen, sauntered across the playing space, and disappeared on the opposite side, all in full view while the two games continued on as before. In the initial preparation of the videotapes, the umbrella-woman event had been chosen simply because, on the face of it, it seemed "odd" in the context of the games. Apparently, subjects agreed with this assessment, since virtually all who did notice her smiled, laughed, or commented spontaneously on her presence. The event was invariably noticed by subjects who were only casually viewing the tape. However, among subjects involved in the main attention task, noticing rates were surprisingly low. Only $21 \%$ of unpracticed subjects showed evidence of noticing when confronted with the odd event on the first trial. The authors investigated the effects of practice on the main task, as well as subjects' expectations regarding the difficulty of the task, manipulated through instructions, and both factors were found to significantly increase noticing rates. They attributed this to an increased ability or propensity of subjects to leave a current "perceptual cycle" (see Neisser, 1976) if they are already skilled on the main task or if, due to instructions, they are led to expect the task not to be overly difficult in the first place. A directed-imagery test, designed to detect possible unconscious influences of the umbrella woman, failed to provide any such evidence in any of the nonnoticing subjects, which is consistent with the hypothesis that the unattended event went genuinely unseen by them.

In the above experiment, the umbrella-woman sequence was preceded as well as followed by about $25 \mathrm{sec}$ of active play in the two superimposed sequences. It is possible that, despite its "oddity," the event was in fact initially noticed by some proportion of subjects classified as "nonnoticers," but was then quickly forgotten due to the demands of the task intervening between the event and the posttrial inquiry. A study by Norman (1969), using a selective-listening technique, found virtually complete forgetting of "unattended," but perceived, digits when the main shadowing task was continued for more than $20 \mathrm{sec}$ after their occurrence. This would seem to lend credence to the idea of rapid forgetting and suggest the necessity of testing this empirically with the umbrella-woman event. One of the main purposes of the present study was to test whether the delay present in the Neisser and Dube (Note 2) experiment decreased noticing rates significantly. In this aim, our study overlapped with a recent experiment by Neisser and Rooney (Note 3). Certain procedural differences between their study and ours make the comparison especially interesting, and a full discussion will therefore be deferred until the results of the present experiment have been presented.

The delay question, referred to above, was directly tested by two of the four experimental groups included in this experiment. The other two groups owed their existence to the serendipitous discovery of a particularly interesting brief segment in the umbrella-woman sequence, a segment that had not been explicitly planned when the tape was originally created. In this segment, the ball in the attended game traveled completely through the body of the umbrella woman and ended up bouncing off the floor right next to her outstretched $\mathrm{leg} /$ foot. Although, in an immediate historical sense she had no part whatsoever in the action in the attended game, the specific frame in question, when viewed as a still picture, unmistakably showed an umbrella-carrying woman kicking a ball to one of the players in the attended game (see Figure 1). Because of this highly meaningful, possible interpretation of this particular image, we reasoned that subjects who saw the tape up to this point might be more likely to incorporate the umbrella woman into their recollection of what they had just seen (if, for example, they were able to inspect the 
immediately preceding "iconic" image; see Neisser, 1967). One group was given the standard posttrial inquiry, and the other was given advance instructions to describe the "last visual image" they had seen just prior to the end of the sequence. It was predicted that such "iconic" instructions would maximize the likelihood of a report of the umbrella woman's presence, if indeed rapid forgetting was the reason for many nonreports.

Finally, we measured the accuracy of subjects' attention performance on the main task prior to, and during, the odd-event sequence. This seemed sensible in light of Neisser and Dube's (Note 2) skill hypothesis, particularly since they never directly measured their subjects' performance levels on the main attention task, but instead based their arguments entirely on the shifts in overall noticing rates that resulted from their experimental manipulations.

\section{METHOD}

\section{Subjects}

Eighty-five undergraduates at Oberlin College participated in the experiment on a volunteer basis.

\section{Apparatus and Stimulus Materials}

The vidcotaped black-and-white stimulus episode was played back on a Panasonic .75-in. cassette recorder (Model NV 9300) and displayed on a 19-in.-diagonal television screen (Sharp Linytron Plus) located at a comfortable viewing distance (a few feet) from the subject's chair. On a table next to the chair was mounted a small microswitch button that the subject pressed in response to the specified target events in the stimulus sequence. The microswitch was connected to a moving-paper event recorder (Esterline Angus), which produced a permanent record of the subject's response pattern. This record was later compared, in the attention-performance scoring procedure, with a specially prepared correct record. The subject operated the microswitch with the preferred hand.

The videotape was a copy of one used in earlier studies (e.g., Neisser \& Dube, Note 2) investigating the noticing of unexpected events in selective looking. The stimulus sequence was a 1-min segment consisting of two independent "basketball games," shown simultaneously in superimposition on the TV screen. Each game featured three men moving irregularly around a room, passing a basektball back and forth among themselves. Each episode contained about 30 passes, but the interthrow intervals were varied unpredictably and feints were occasionally introduced to ensure that good attention performance would necessitate close monitoring of the screen. The two superimposed games showed the same individuals playing the same kind of game, although the games had been recorded separately. In one game, the players wore black shirts, and in the other, white shirts; this provided a highly visible, and typical, cue for telling the games apart. What was unusual, of course, was the effect of the superimposition: Players from different games walked "through" one another in a ghost-like fashion whenever they overlapped on the screen, whereas within a game the normal rules of occlusion held.

The episode started with the appearance of the three blackshirted players, each bouncing the ball twice off the floor to provide synchronization signals for the event recorder (the experimenter activated the microswitch for each bounce). After 3 sec of blank screen, the black-shirted players reappeared, now playing the passing game described above. Seven seconds later, the white-shirted players gradually faded in, until both games were about equally salient in terms of physical visibility. The games continued, in superimposition, for the remainder of the minute.
Twenty-five seconds after the fade-in, the unexpected target event was introduced into the game with the white-shirted players. A woman carrying a large white umbrella (the "umbrella woman," or U.W.) that partially obscured her head from the camera entered the field of view and walked in a leisurely fashion from left to right across the screen between the camera and the players. She never participated in the game in any way. Her on-screen presence lasted a total of $5.5 \mathrm{sec}$, during which time she took eight steps.

In the main attention task, the subjects always monitored the black-shirted players. Since the superimpositioning had been accomplished electronically prior to the experiment, U.W. always entered the unattended game in exactly the same spot relative to the action in the attended game. Her first on-screen step occurred just as a pass thrown through the air reached player R.B.; at this point, the images of U.W. and R.B. were perfectly superimposed. During U.W.'s second step, R.B. turned around and prepared for a bounce pass to player D.L. As the pass left R.B.'s hands, it traveled through U.W.'s umbrella, at the approximate height of her head, and continued downward through her torso, hips, and leg. Meanwhile, U.W. prepared for and took her third on-screen step. Coincidentally, the (attended) ball and the L.W.'s (unattended) left foot reached the floor at the identical location at the identical time. The frame immediately following on the videotape gave the distinct impression of an umbrellacarring person playing soccer (see Figure 1 for a close rendering of the stimulus tape at the point of the "soccer-kick" frame). After D.L. received the pass, he bounced it on the floor several times, while his teammates moved to new locations in the room. He then turned around and passed it across the room, away from the camera, to R.B. During D.L.'s dribbling performance, U.W. continued toward the right side of the screen, walking right "through" D.L. on the way. She disappeared from the field of view on the right side of the screen, just as D.L's pass was in midair (the "exit pass"). U.W.'s projected size varied between $68 \%$ of the screen size vertically and $20 \%$ horizontally at the left side, and $46 \%$ vertically, $11 \%$ horizontally, at the right side, the decrease being due to the fact that she walked diagonally across the room, away from the camera. During, as well as following, the U.W. sequence, the two games were played in the same manner as before her appearance.

Figure 2 shows the time relations among various important features of the U.W. target sequence. As can be seen, the action in the attended game was relatively dense before the "soccer kick," with two passes occurring within a short period of time. After the "soccer kick," however, about 3 sec elapsed before the next pass occurred, and during this period, D.L., facing the camera and dribbling, was in no position to pass until he turned

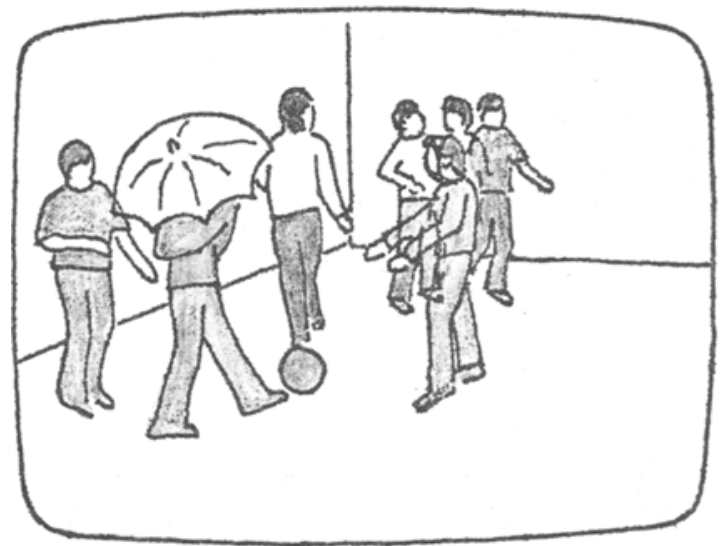

Figure 1. Appearance of the stimulus tape at the point of the "soccer-kick" frame. 


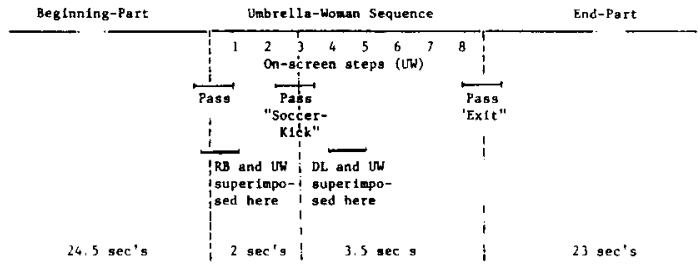
each Treatment Group

Ex

\& $x$

Figure 2. Top: Schematic outline of the stimulus sequence, showing temporal relationships among events during the umbreila-woman sequence. Bottom: Exposure for each treatment group.

around and found one of his teammates, both of whom were at the far end of the playing space. U.W. had time for five steps before the pass was released. Direct overlap between U.W. and a ball-handling player in the attended game occurred during steps 1 and 2 (with R.B.) and steps 4 and 5 (with D.L.).

\section{Procedure}

All subjects were tested individually. After being shown where to sit and how to operate the response button, the subjects were told that when the tape started they would first see the dark-shirted players briefly, followed by a blank, and then the start of the "game." They were instructed to follow closely the action in the game with the dark-shirted players and to press the button as soon as a pass occurred; they were warned that there would be feints and that a response in such an instance would constitute an error, as would a late response.

The subjects were then informed that, after a few seconds of play, a second team, playing in the same manner as the first, but wearing white instead of black shirts, would gradually fade in on top of the game they were following. They were told to keep following the black-shirted players to the end of the trial, despite the superimposition of the other players; they were warned that the task would be rather difficult.

The above instructions were given to all groups prior to the task. Certain additional instructions given to the "kick-icon" group are described below. The instructions were entirely verbal; no hands-on practice was given and no mention of the umbrella woman was made to any of the subjects before the experimental trial.

The subjects were divided into four experimental groups designated "total," "exit," "kick," and "kick icon" (abbreviated $\mathrm{T}, \mathrm{E}, \mathrm{K}$, and $\mathrm{KI}$, respectively). The first three groups, comprising 66 randomly assigned subjects, participated in the initial experiment. The groups differed in the amount of material they saw after the entrance of U.W. Group T viewed the entire 1-min videotape, Group E saw the first $30 \mathrm{sec}$, up to the point of U.W.'s exit, and Group $K$ viewed the tape for $26.5 \mathrm{sec}$ after the start of the sequence, up to the "soccer-kick" frame (described above and shown in Figure 1).

The last 20 subjects (Condition $\mathrm{KI}$ ) were shown the same tape as the subjects in Condition $K$ and were, in addition, given the following "iconic" instructions. Prior to being shown the sequence, they were told that the tape would end suddenly, with the screen going blank right in the middle of play. They were asked to then attempt to remember the "last visual image" presented on the screen before the disappearance, a task that, the experimenter advised would be analogous to working with the afterimage of the final videotape frame. This would be similar, the experimenter said, to seeing the image left when a light that one is looking at goes out. To illustrate this, the subjects were told to look toward the neon ceiling lights in the experimental room as the experimenter flicked them off for a few seconds. They were instructed to begin describing the last image immediately after the tape ended.

There were three ways of determining whether or not a given subject noticed U.W. First, the experimenter watched his or her reactions during the sequence with U.W. As had been observed in previous experiments using this tape, almost all subjects who do notice U.W. smile, laugh, or make some type of verbal exclamation upon becoming aware of her presence, and our subjects were no exception to this rule. Second, the experimenter asked a series of probing questions after the showing of the tape: "Was that difficult? Were there any particularly hard places? Was there anything especially worth noting in the episode?" If the subject still gave no indication of having seen U.W., the critical tape segment was shown again and the subject was asked if he or she remembered seeing her but had just failed to mention it. If, at this point, the subject still showed no indication of recognition of U.W., he or she was recorded as a "nonnoticer."

\section{RESULTS}

The two major dependent measures collected for each subject consisted of the binary classification into the "noticer" and "nonnoticer" groups and the sequence of responses to passes in the main target game. Each is discussed in turn, and then they are compared.

\section{U.W.-Noticing Rates}

The proportion of subjects who noticed U.W. differed significantly among the treatment groups $\left[\chi^{2}(3)=9.3\right.$, $\mathrm{p}<.03]$. Table 1 presents raw data, as well as percentages. It is evident from visual inspection that the crucial variable determining the likelihood of noticing was the amount of the total U.W. sequence that the subjects had seen rather than the delay, or the amount of activity, between the end of the U.W. sequence and the report. The results for Groups $\mathrm{T}$ and $\mathrm{E}$ are virtually identical and are also in good agreement with previously reported findings (e.g., Neisser \& Dube, Note 2). Similarly, the results for Groups $\mathrm{K}$ and $\mathrm{KI}$ are almost the same. It is convenient, therefore, to combine Groups $T$ and $\mathrm{E}$ into a FULL (i.e., exposure to the full U.W. sequence) and Groups $\mathrm{K}$ and $\mathrm{KI}$ into a PARTIAL (i.e., exposure to the partial U.W. sequence) group. Analyzed in this fashion, the FULL group exhibited a $34 \%$ noticing rate, as against a $7 \%$ rate for the PARTIAL group. These differences are highly significant $(z=3.02$, $\mathrm{p}<.01)$.

Table 1

Odd-Event Noticing Rates in the Four Treatment Groups

\begin{tabular}{lcccc}
\hline & \multicolumn{4}{c}{ Treatment Groups } \\
\cline { 2 - 5 } & Total & Exit & Kick & Kick Icon \\
\hline Noticers & 8 & 7 & 1 & 2 \\
Nonnoticers & 15 & 14 & 20 & 18 \\
Total & 23 & 21 & 21 & 20 \\
Percent Noticing & 35 & 33 & 5 & 10 \\
\hline
\end{tabular}


Following Performance Prior to U.W. Sequence

Event records were scored for all groups from the beginning up until the U.W. sequence. The scoring in volved comparing each subject's response record with the master target record and deriving an index of performance that reflected the degree of coincidence between the two. To this end, we used the following method: A "hit" was scored whenever a response was made at any time within an interval spanning $.25 \mathrm{sec}$ before to $.75 \mathrm{sec}$ after a pass in the target game. Responses elsewhere ("false alarms") and absences of responses in pass areas ("misses") were simply scored as "errors." At any given time, the subject was considered to be either "on target" or "off target." Between hits, he or she was scored as "on," and between errors, as "off," for the entire period. Between a hit and an error, the record was scored as "on" from the hit until halfway between the hit and the error, at which point it was scored as "off" up until the error. With this method, a subject's performance could be expressed in terms of the proportion of time of the total sequence, during which he or she was "on target." This fraction is referred to as the raw "on-time" (ROT) index.

The ROT index is necessarily an inflated index, as long as subjects respond at all, since even totally random performance will result in a nonzero fraction. We therefore used a corrected "on-time" (COT) index, devised by one of us (R.B.) in collaboration with Ulric Neisser, which utilizes the distribution of probabilities of finding "on-time" runs, of various lengths, by chance alone. ${ }^{1}$ With the present target and response probabilities, it can be shown that $95 \%$ of all such chance runs will be less than $6 \mathrm{sec}$ long. The COT index, then, represents the "on-time" fraction derived from the sum of all runs spanning $6 \mathrm{sec}$ or more.

In general, the subjects performed well in following the target game and reported that the task was not overly difficult. Table 2 gives mean COT scores for "noticers" and "nonnoticers" within each treatment group. Note that these scores represent performance prior to any treatment separation and therefore ought not to differ due to treatments. When collapsed across the dependent "noticing" variable, the means do not, in fact, differ $[F(3,81)=1.41$, n.s. $]$. Visual inspection of the means suggests, however, that interactions paralleling those found for noticing rates are present in the following-

Table 2

Following Performance Prior to the Umbrella-Woman Sequence, as Measured by COT Scores (Perfect Performance $\mathbf{~ 1 ) , ~ f o r ~ t h e ~ F o u r ~}$ Separate Treatments

\begin{tabular}{lccccc}
\cline { 5 - 6 } & \multicolumn{4}{c}{ Treatment Groups } \\
\cline { 1 - 1 } \cline { 5 - 6 } & Total & & Exit & Kick & Kick Icon \\
\cline { 1 - 2 } Noticers & .89 & & .80 & .46 & .70 \\
Nonnoticers & .85 & .87 & .80 & .88 \\
Weighted Means & .86 & .85 & .78 & .86 \\
\hline
\end{tabular}

Table 3

Following Performance Prior to Umbrella-Woman Sequence, as Measured by COT Scores, for FULL Exposure (Groups "Total" and "Exit") and PARTIAL

Exposure (Groups "Kick" and "Kick Icon") Treatments

\begin{tabular}{|c|c|c|c|c|c|c|}
\hline & & \multicolumn{2}{|l|}{ FULL } & \multicolumn{3}{|c|}{ PARTIAL } \\
\hline & M & SD & $\mathrm{N}$ & M & SD & $\mathrm{N}$ \\
\hline Noticers & .85 & .14 & 15 & .62 & .16 & 3 \\
\hline Nonnoticers & .86 & .16 & 29 & .83 & .14 & 38 \\
\hline Weighted Total & .86 & .15 & 44 & .82 & .15 & 41 \\
\hline
\end{tabular}

Note $-M=$ mean $; S D=$ standard deviations; and $N=$ number of subjects.

performance data as well. The extreme variations in group size make a comprehensive ANOVA impossible. However, if noticers and nonnoticers are compared within each treatment group separately, adequate justification is found for increasing power by combining the groups into FULL and PARTIAL conditions, as was done with the noticing-rate data [noticers vs. nonnoticers, Group A: $\mathrm{t}(21)=.60$, n.s.; Group B: $\mathrm{t}(19)=$ .94 , n.s.; Group $\mathrm{C} 1: \mathrm{z}=2.12, \mathrm{p}<.02^{2}$; Group C2: $t(18)=2.41, p<.03]$. The combined data appear in Table 3 .

The overall FULL vs. PARTIAL comparison is not intrinsically meaningful, since the treatments did not differ during the period represented by the data. In conjunction with the dependent "noticer" variable, however, the comparison is of interest. Testing for the specific interaction in the PARTIAL/noticers cell, by means of a $t$ test for contrasts, reveals that these subjects were indeed inferior in their following performance on the main task even prior to the appearance of U.W. $\left[t_{\psi}(81)=2.42, p<.02\right]$. There are no other significant differences in Table 3 .

\section{Following Performance During the U.W Sequence}

It is of interest to compare the attention performance of noticers and nonnoticers during the appearance of U.W. The FULL condition featured two on-screen passes with U.W. clearly in view (the "soccer-kick" pass and the "exit" pass), whereas the PARITAL condition included only the first of these. Table 4 shows how many subjects responded properly to these throws for each of the treatments. There is no evidence of differences between noticers and nonnoticers in the FULL group; the "soccer-kick" pass appears to have been slightly more difficult for everyone than the "exit" pass, but this was equally true for both types of subjects. In the PARITAL group, the pattern is different: All of the noticers missed the "soccer-kick" pass, whereas only $32 \%$ of the nonnoticers failed to respond to this pass. The probability of this result may be estimated by means of the hypergeometric distribution: If a population consisting of 26 "on-target" and 15 "off-target" subjects is sampled three times in succession, without re- 
Table 4

Following Performance During the Unexpected-Event Sequence in Terms of the Number of Subjects Who Successfully Responded to Each of the Two On-Screen Passes That Occurred During the Umbrella-Woman Sequence

\begin{tabular}{|c|c|c|c|c|c|}
\hline & \multirow[b]{2}{*}{$\begin{array}{c}\text { Total } \\
\mathbf{N}\end{array}$} & \multicolumn{2}{|c|}{ "Soccer-Kick" Pass } & \multicolumn{2}{|c|}{ "Exit" Pass } \\
\hline & & $\begin{array}{l}\text { No. "On- } \\
\text { Target" }\end{array}$ & $\begin{array}{l}\text { Per- } \\
\text { cent }\end{array}$ & $\begin{array}{l}\text { No. "On- } \\
\text { Target" }\end{array}$ & $\begin{array}{l}\text { Per- } \\
\text { cent }\end{array}$ \\
\hline \multicolumn{6}{|c|}{ FULL } \\
\hline Noticers & 15 & 13 & 87 & 15 & 100 \\
\hline Nonnoticers & 29 & 25 & 86 & 28 & 97 \\
\hline \multicolumn{6}{|c|}{ PARTIAL } \\
\hline Noticers & 3 & 0 & 0 & & \\
\hline Nonnoticers & 38 & 26 & 68 & & \\
\hline
\end{tabular}

Note-Subjects in the PARTIAL group did not see the "exit" pass.

placement, the probability of randomly choosing three "off-target" subjects equals .043. Despite the small number of subjects in the PARTIAL/noticer group, it seems prudent to conclude that these subjects missed the "soccer-kick" pass more often than one would expect on the basis of chance alone.

Since only half of the "soccer-kick" pass was actually shown to the subjects in the PARTIAL treatment, the performance discrepancy between the PARTIAL/ nonnoticers and the subjects in the FULL treatment on that pass was most likely simply a reflection of this difference in the stimulus situation.

\section{DISCUSSION}

With respect to the questions that initially motivated this experiment, the results of the study are clear. There was no evidence that $23 \mathrm{sec}$ of selective looking after the unexpected target event affected noticing rates one way or the other: Subjects either noticed and remembered the umbrella woman until the end of the trial or failed to do either. Delay of the posttrial inquiry made no difference. Noticing rates among subjects who saw the FULL umbrella-woman sequence are consistent with the results of previous studies. Neisser and Dube's (Note 2) finding of $21 \%$ is not significantly different from the present experiment's $35 \%$ and $33 \%$ for Groups $T$ and $E$, respectively $\left[\chi^{2}(2)=1.34, n . s.\right]$. With an umbrellawoman sequence created on a different occasion, but in the same fashion as ours, Neisser and Rooney (Note 3; also described below) estimated a $30 \%$ true noticing rate with a forced-choice technique. Neisser, Dube, Karis, and Bahrick (as reported by Neisser \& Rooney, Note 3) found a noticing rate of $25 \%$ with adult subjects, and Neisser and Rooney (cited in Neisser, 1979) found a $22 \%$ rate with fourth-grade children. The expectation for first-trial noticing for subjects who see the kind of umbrella-woman sequence used in these experiments appears to lie between $20 \%$ and $35 \%$.
A comparison of noticing rates for Groups $\mathrm{K}$ and $\mathrm{KI}$ leads to a similar conclusion: The delay involved in conducting the posttrial inquiry can hardly be said to have led to the "fading" of some brief form of memory for the umbrella-woman event, since when subjects were instructed in advance to describe, in detail, the "last visual image" prior to the expected interruption, noticing rates did not increase significantly. The subjects in these groups either saw and reported on her appearance or missed her altogether.

The strong differences in noticing rates that were found in this study become evident when subjects who saw the FULL umbrella-woman sequence (Groups T and E) are compared with those who saw only the PARTIAL sequence, up to the "soccer-kick" pass (Groups K and KI). The "iconic"-instruction group (KI) showed surprisingly low noticing in light of what, from one point of view, might be considered nearly optimal stimulus conditions for seeing the umbrella woman: The ball went right through her entire body, which is probably where most subjects foveated at the time. In addition, her position in the last frame was such that she could quite easily have been seen as an integral part of the attended game itself, if what subjects did was to scan an iconic image. Yet, hardly anyone saw her.

It could be hypothesized that the very low rate of noticing in the PARTIAL groups was due to some kind of backward-masking process (Kahneman, 1968), because of the visual characteristics of the postexposure field. Such an account, however, is highly unlikely. More than half of the nonnoticers in the "iconic"-instructions group produced painstakingly accurate and detailed descriptions of the exact positions of the players and the ball in the attended game, a number of them even going so far as to trace the precise trajectory of the ball during the "soccer-kick" pass, directly on the screen. Most subjects were able to give a very exact description of the action in the attended game (occurring at the left part of the screen) without mentioning the most (physically) prominent object present at that location, in the actual image. If the postexposure field somehow masked the immediately preceding image, attended as well as unattended materials ought to have been similarly affected, but this was clearly not the case. More seriously, it is possible that the very strong tendency of our subjects to focus their descriptions on the action in the attended game reflected a response bias that, in effect, prevented the umbrella woman's being reported before the fleeting memory of her presence had already faded into oblivion. Although our experiment does not permit a direct evaluation of this idea, Neisser and Rooney's (Note 3) adoption of a forced-choice methodology would seem to eliminate such reporting bias as a factor. Their subjects viewed a tape similar to ours, one that also featured an unexpected umbrella woman. About two-thirds through the umbrella-woman sequence in their experiment, a split screen suddenly appeared, with an umbrella woman on one side (in a different pose from the one assumed 
by the umbrella woman who had just walked by) and a young boy holding a can of soda on the other. Subjects had to choose immediately which of the two had just appeared on the screen, moments earlier. After correcting for chance, the authors estimated a $30 \%$ noticing rate, which was identical to the rate obtained with the same videotape when the standard postquestioning procedures were used (Neisser, Dube, Karis, \& Bahrick, as cited in Neisser \& Rooney, Note 3). Taken together, these experiments suggest that the umbrella woman went genuinely unnoticed by subjects who failed to report her. "Late"-selection theories receive no support from our data.

It might be argued that noticing in our experiment was simply a function of length of exposure to the unexpected event. One could, for example, construe a filter theory in which sampling of the information in "unattended channels" proceeds according to a certain rate of filter-criterion switching with some fixed probability of noticing during each switch (see Moray, 1970 , for a similar argument). If so, the longer the on-screen presence of the unexpected target event is, the greater the likelihood of noticing. The most straightforward prediction would be a simple linear relationship between time and noticing probability. In our experiment, the FULL group was in fact exposed to the umbrella woman for a longer period of time, and reported on her presence more often, than did the PARTIAL group, just as would be predicted by a "filter-criterion-switching" hypothesis. Quantitatively, our figures turn out to be quite inconsistent with such a notion, however. Only about onefifth $(7 / 34)$ of the total noticing occurred in more than one-third (2/5.5) of the total time, and, as we shall see, among skilled subjects, nobody noticed during the first third of the umbrella-woman sequence. The switching of attention that may well occur is not conveniently described in terms of a mechanical-like sampling hypothesis, but is better understood by reference to the specific interactions occurring at each moment in the attended game. Skilled perceivers are able to take advantage of anticipatory opportunities and will gauge their exploratory information pickup activities accordingly. That most noticing would take place after the "soccer-kick" pass agrees well with what one would predict from an analysis of the sequence itself (see Apparatus and Stimulus Materials section; also see Figure 2); Player D.L. and the umbrella woman are initially superimposed, and D.L. is out of position to pass the ball until he turns around. To use this information, a subject not only has to know where the ball is, but also where the players are in relation to one another, in a gestalt sense; a skilled perceiver keeps track, not only of the ball, but also of the configuration of the entire game from moment to moment. During times of immediate preparation for passing, or in the middle of a throw, these subjects wisely restrict their engagement in subsidiary exploratory cycles, since, after all, their primary task is to push the button during, or soon after, a pass. The superior hit rates for noticers as well as nonnoticers in the FULL group on the "exit" pass as opposed to the "soccerkick" pass shows empirically that the latter part of the umbrella-woman sequence was in fact less demanding and presumably therefore was exploited by skilled subjects for perceptual exploration. The same point can also be made by selecting subjects according to a skill criterion on the COT scores. If subjects below $80 \%$ "on-target" time (the lowest mean among the nonnoticer groups) are eliminated from all groups, FULL-group noticing remains at $30 \%(9 / 30)$, whereas PARTIAL-group noticing drops to $0 \%$. Skilled subjects who noticed appear to have done so precisely when the potential for anticipation was highest.

Why, it is reasonable to ask, did a few subjects actually notice the umbrella woman prior to the "soccerkick" pass, when, by our previous argument, the attended game action was difficult and the anticipatory opportunities were few? As has already been intimated, our procedures appear to have identified two subgroups of noticers, who saw the unexpected event for very different reasons indeed. The argument rests on the finding that the PARTIAL noticers were inferior overall on the main attention task prior to, as well as during, the umbrella-woman sequence. Their COT scores were significantly lower than those of other groups, and they all failed to respond to the last pass, an unlikely event if they came from the same statistical population as PARTIAL nonnoticers. It seems that, despite instructions that placed emphasis on performing well on the main following task, these few subjects (and probably a few among the FULL noticers as well) were significantly poorer at this than the rest, and they were in fact the very ones to notice the umbrella woman under adverse conditions. These subjects were probably more prone throughout than the rest to engage in exploratory perceptual cycles, but they paid a price for their curiositythey missed the main target instead!

These results confirm what is implicit in Neisser and Dube's (Note 2) findings on the effects of practice and instructions on noticing rates. Both of these variables, they showed, could boost noticing rates substantially, although their discussion focused primarily on the attentional skills presumably developed by the practiced noticers. Our results suggest that practice and instructions accomplish two different things entirely, both of which, however, favor noticing. Skill permits subjects to discover, and make use of, the overall anticipatory structure of the main game so that they may distribute their exploratory activities optimally, according to that structure. With increased practice, subjects can thus be expected to learn more about unattended materials, provided they are inclined to engage in secondary cycles in the first place (alternatively, they may use the time to explore aspects of their own inner activities, such as daydreaming, menu planning, etc.). In any case, attended game performance need not suffer from such activities. In contrast, instructions are likely to shift subjects' 
priorities, rather than evoke more skillful behavior, and this should result in loss of accuracy on the attended game but a greater overall noticing probability, due to the increased explorations. Had Neisser and Dube (Note 2) actually measured performance levels on the main task, we believe they would have found such a tradeoff between accuracy and noticing rates in the instruction conditions, but not in the practice conditions.

\section{REFERENCE NOTES}

1. Becklen, R., Neisser, U., \& Littman, D. The effect of event similarity on selective looking. Manuscript in preparation, 1983.

2. Neisser, U., \& Dube, E. F. Interrupting the perceptual cycle: When do we notice unexpected events? Paper presented at the meeting of the Eastern Psychological Association, Washington, D.C., March 1978.

3. Neisser, U., \& Rooney, P. Noticing unexpected events in selective looking: $A$ new criterion. Unpublished manuscript, 1982.

4. Becklen, R. A method for determining percent on-target time in continuous attention experiments with varying target- and response-probabilities. Unpublished manuscript, 1983.

\section{REFERENCES}

Broadbent, D. E. Perception and communication. New York: Pergamon Press, 1958.

Corteen, R. S., \& Wood, B. Autonomic responses to shockassociated words in an unattended channel. Journal of Experimental Psychology, 1972, 94, 308-313.

Deutsch, J. A., \& Deutsch, D. Attention: Some theoretical considerations. Psychological Review, 1963, 70, 80-90.

Kahneman, D. Methods, findings and theory in studies of visual masking. Psychological Bulletin, 1968, 70, 404-425.

Kahneman, D. Attention and effort. Englewood Cliffs, N.J: Prentice-Hall, 1973.

Mackay, D. G. Aspects of the theory of comprehension, memory and attention. Quarterly Journal of Experimental Psychology, 1973, 25, 22-40.

Moray, N. Attention in dichotic listening: Affective cues and the influence of instructions. Quarterly Journal of Experimental Psychology, 1959, 11, 56-60.

Moray, N. Attention: Selective processes in vision and hearing. New York: Academic Press, 1970.

Nersser, U. Cognitive psychology. New York: Appleton-CenturyCrofts, 1967.

Neisser, U. Cognition and reality. San Francisco: Freeman, 1976.

Nersser, U. The control of information pickup in selective looking. In H. Pick (Ed.), Perception and development: $A$ tribute to Eleanor Gibson. New York: Halsted Press, 1979.

Neisser, U., BeckLEN, R. Selective looking: Attending to visually specified events. Cognitive Psychology, 1975, 7, 480-494.

Norman, D. A. Toward a theory of memory and attention. Psychological Review, 1968, 75, 522-536.

Norman, D. A. Memory while shadowing. Quarterly Journal of Experimental Psychology, 1969, 21, 85-93.
Norman, D. A. Memory and attention. New York: Wiley, 1976.

Spelke, E. S., Hirst, W. C., \& Neisse R, U. Skills of divided attention. Cognition, 1976, 4, 215-230.

von Wrioht, J. M., Anderson, K., \& Stenman, U. Generalization of conditioned GSR's in dichotic listening. In P. M. A. Rabbitt \& S. Dornic (Eds.), Attention and performance $V$. New York: Academic Press, 1975.

Wardlaw, K. A., \& Kroll, N. E. A. Autonomic responses to shock-associated words in a non-attended message: $A$ failure to replicate. Journal of Experimental Psychology: Human Perception and Performance, 1976, 2, 357-360.

\section{NOTES}

1. Calculations of chance on-target rates are based on the forlowing assumptions: The total trial is divided up into a string of fixed-length criterion-interval slots, in which targets and responses occur independently and randomly with probabilities $p(t)$ and $p(r)$, respectively. Any random interval will then contain one of the following joint events:

$$
\begin{aligned}
& \mathrm{H}: \text { "Hit" }=\text { target, response. } \mathrm{p}(\mathrm{H})=\mathrm{p}(\mathrm{t}) \cdot \mathrm{p}(\mathrm{r}) \text {. } \\
& \mathrm{E}: \text { "Error" }=\text { target, no response or no target, response. } \\
& \mathrm{p}(\mathrm{E})=\mathrm{p}(\mathrm{t}) \cdot[1-\mathrm{p}(\mathrm{r})]+[1-\mathrm{p}(\mathrm{t})] \cdot \mathrm{p}(\mathrm{r}) . \\
& \mathrm{N}: \text { "Null" }=\text { no target, no response. } \mathrm{p}(\mathrm{N})=[1-\mathrm{p}(\mathrm{t})] \cdot \\
& {[1-\mathrm{p}(\mathrm{r})] .}
\end{aligned}
$$

On the basis of the scoring rules described in the text, "ontarget" runs will consist of strings of adjacent intervals of $\mathrm{Hs}$ and Ns. The cumulative probability distribution for obtaining such strings of length $m$ or less $(m \geqslant 1)$ has been shown (Becklen, Note 4) to equal:

$p(E)^{2} \cdot \sum_{n=2}^{2 m} \sum_{i=1}^{l} \sum_{j=0}^{r}(i-j) \cdot(n-2 i+1)$

$$
\begin{gathered}
\left(\begin{array}{c}
r \\
j
\end{array}\right) \cdot p(H)^{(i-j-1)} \cdot p(N)^{(n-2 i+j)}, \\
{\left[\begin{array}{l}
r=0 \text { for } i=1 \\
r=i-2 \text { for } i>1
\end{array}\right] ;\left[\begin{array}{l}
\ell=n / 2 \text { for even } n s \\
l=(n-1) / 2 \text { for odd } n s
\end{array}\right] .}
\end{gathered}
$$

where

In the present experiment, $p(t)$ and $p(r)$ were empirically determined from the stimulus and response tapes to equal approximately $1 / 2$. This yields a probability of about .05 for chance runs of $6 \mathrm{sec}$ or more, which was used as our COT scoring criterion.

2 . Probability that a score, picked at random from an assumed normal distribution with $\mu=.80$ and $\sigma=.16$ (empirical values for Group $K$, nonnoticers) will deviate as much as or more than the single noticer score (.46).

(Manuscript received February 3, 1983; revision accepted for publication August 23, 1983.) 